\title{
Altas dosis de quimioterapia y soporte hematopoyético en el tratamiento de niños con meduloblastoma y tumor neuroectodérmico primitivo supratentorial de alto riesgo
}

\author{
A. Pérez Martínez, V. Quintero Calcaño, M. González Vicent, J. Sevilla Navarro, M. A. Díaz Pérez, \\ L. Madero López
}

\section{Resumen}

- Propósito: las altas dosis de quimioterapia (ADQ) y el rescate con progenitores hematopoyéticos autólogos ha demostrado mejorar la supervivencia en pacientes con meduloblastoma (MB) y tumor neuroectodérmico primitivo supratentorial (stPNET) recurrente y de alto riesgo.

- Material y métodos: presentamos 19 pacientes tratados con $A D Q, 13$ de alto riesgo y 6 con enfermedad recurrente. Los pacientes fueron movilizados con factores estimulantes de colonias granulocíticas (G-CSF) a dosis de $12 \mu \mathrm{g} / \mathrm{kg} / 12 \mathrm{~h}$ durante 4 días. El acondicionamiento consistió en busulfán-melfalán. Tres pacientes recibieron de manera adicional tiotepa y cuatro pacientes topotecán. Los progenitores hematopoyéticos fueron reinfundidos $48 \mathrm{~h}$ tras finalizar la quimioterapia.

- Resultados: con una mediana de seguimiento de 18 meses (rango 5-63) tras el trasplante, 9 pacientes (47\%) están vivos ( 8 en remisión completa y 1 en remisión parcial). Fallecieron 3 pacientes (15\%) por toxicidad del procedimiento y 7 por enfermedad progresiva (36\%). La supervivencia libre de eventos, según el método de Kaplan-Meier, es del $37,67 \pm 14 \%$ en todos los pacientes y un $57 \pm 15 \%$ en los pacientes de alto riesgo.

- Conclusiones: en nuestra experiencia las $A D Q$, aunque es un procedimiento tóxico, puede mejorar la supervivencia especialmente en pacientes con MB o stPNET de alto riesgo.

\section{Palabras clave:}

Trasplante autólogo de progenitores hematopoyéticos. Altas dosis de quimioterapia. Meduloblastoma. Tumores neuroectodérmicos primitivos supratentoriales.

Oncología, 2004; 27 (2):60-68 


\section{Summary}

- Purpose: The combination of high-dose chemotherapy (HDC) and autologous stem cells rescue (ASCR) has been reported as a new relevant treatment improving survival rates of patients with cerebellar medulloblastoma and supratentorial neuroectodermal tumors. We report our preliminary results employing the procedure.

- Material and methods: We study 19 patients treated with HDC and ASCR; 13 of them were high risk patients, and 6 had recurrent disease. The patients received granulocyte colony-stimulating factor (G-CSF) at a dose of $12 \mu \mathrm{g} / \mathrm{kg} / 12 \mathrm{~h}$ during four days to stimulate granulocytes proliferation and mobilization. The patients were conditioned with busulfan-melphalan. Additionally, thiotepa was administered to three patients, and topotecan to four. The peripheral blood progenitor cells were collected and infused $48 \mathrm{~h}$ after finishing chemotherapy.

- Results: In an average follow-up of 18 months (range 5-63 months) after transplantation, 9 patients $(47 \%)$ survived disease-free, 8 with complete remission and 1 with partial remission. Three patients $(15 \%)$ died due to the toxic effects of the treatment, and $7(36 \%)$ because of disease progression. According to the Kaplan-Meier method, the disease-free survivors were $37.67 \pm 14 \%$ as a whole, and $57 \pm 15 \%$ for the high risk patients.

- Conclusions: Although the combined use of high-dose chemotherapy and autologous hemopoietic stem cells transplantation is a toxic treatment, in our experience it improves the survival of patients with high risk medulloblastoma and supratentorial primitive neuroectodermal tumors, especially of the later.

Key words: Peripheral blood progenitor cells. Autologous transplantation. High-dose chemotherapy. High-risk brain tumors. Medulloblastoma. Supratentorial primitive neuroectodermal tumors.

\section{Introducción}

El tratamiento multidisciplinario con cirugía, radioterapia y quimioterapia ha permitido mejorar la supervivencia global de los pacientes con meduloblastomas (MB) y tumores neuroectodérmicos primitivos supratentoriales (stPNET), hasta situarla actualmente alrededor del $50-70 \%$.

Sin embargo, los pacientes considerados de alto riesgo y los que presentan enfermedad recurrente, tienen un pronóstico muy desfavorable $e^{2,3}$.

En los últimos años y con el objetivo de mejorar la supervivencia en este grupo de pacientes, se han realizado diversas alternativas terapéuticas ${ }^{1,4}$. Una de estas estrategias la constituye la intensificación del tratamiento con altas dosis de quimioterapia $(A D Q)$ y el rescate con progenitores hematopoyéticos autólogos.

Los resultados obtenidos por los diferentes grupos son actualmente objeto de debate y revisión en numerosos artículos científicos ${ }^{4,5}$.

En este artículo, presentamos nuestra experiencia con altas dosis de quimioterapia y rescate con progenitores hematopoyéticos autólogos de sangre periféri$\mathrm{ca}$, en el tratamiento del MB y stPNET en pacientes de alto riesgo o en enfermedad recurrente.

\section{Material y métodos}

\section{- Pacientes}

Entre marzo 1995 y diciembre 2002, 19 pacientes pediátricos con diagnóstico histológico de $M B \circ$ stPNET fueron sometidos a altas dosis de quimioterapia y rescate con progenitores hematopoyéticos autólogos.

Diferenciamos dos grupos de pacientes: por un lado los de alto riesgo y por otro aquellos que presentaban enfermedad recurrente.

\section{- Pacientes de alto riesgo}

El tratamiento inicial en los pacientes de alto riesgo consistió en resección tumoral, radioterapia en niños mayores de 4 años y quimioterapia estándar.

En todos los pacientes se intentó la máxima resección de la tumoración.

Las dosis de radioterapia fueron 50 Gy sobre el tumor primario, durante 6-7 semanas en fracciones diarias de $2 \mathrm{~Gy}$ (de lunes a viernes, descansando el fin de semana) y 35 Gy sobre el eje craneoespinal.

Los regímenes de quimioterapia estándar fueron los protocolos de quimioterapia de la Sociedad Francesa de Oncología Pediátrica (SFOP) 6 , Sociedad Internacio- 


\section{A. Pérez Martínez y cols.}

TABLA I

Características de los pacientes de alto riesgo

\begin{tabular}{|c|c|c|c|c|c|c|c|c|c|}
\hline & Sexo & Edad & Diagnóstico & Cirugía & Radioterapia & $\begin{array}{l}\text { Quimioterapia } \\
\text { estándar }\end{array}$ & $\begin{array}{l}\text { Situación } \\
\text { clínica }\end{array}$ & Chang & $\begin{array}{l}\text { Meses desde } \\
\text { el diagnóstico } \\
\text { al trasplante }\end{array}$ \\
\hline Paciente 1 & Niño & 2 & $M B$ & Parcial & $\mathrm{NO}$ & $\mathrm{NO}$ & EP & $\mathrm{T} 4$ & 2 \\
\hline Paciente 2 & Niño & 4 & $M B$ & Total & $\mathrm{NO}$ & $\mathrm{NO}$ & $\mathrm{RC}$ & T1 & 1 \\
\hline Paciente 3 & Niño & 2 & stPNET & Total & $\mathrm{NO}$ & SFOP & $\mathrm{RC}$ & $\mathrm{Tl}$ & 3 \\
\hline Paciente 4 & Niña & 2 & stPNET & Total & NO & NO & $\mathrm{RP}$ & $\mathrm{Tl}$ & 2 \\
\hline Paciente 5 & Niña & 14 & $M B$ & Parcial & sí & CCG & $\mathrm{RP}$ & $\mathrm{Tl}$ & 2 \\
\hline Paciente 6 & Niño & 2 & $M B$ & Total & NO & SIOP & $\mathrm{RC}$ & $\mathrm{Tl}$ & 4 \\
\hline Paciente 7 & Niño & 1 & $M B$ & Total & NO & SIOP & $\mathrm{RC}$ & $\mathrm{Tl}$ & 5 \\
\hline Paciente 8 & Niña & 5 & stPNET & Total & sí & NO & $\mathrm{RC}$ & $\mathrm{Tl}$ & 6 \\
\hline Paciente 9 & Niña & 9 & $M B$ & Subtotal & Sí & NO & $\mathrm{RC}$ & $\mathrm{T} 1$ & 7 \\
\hline Paciente 10 & Niña & 2 & $M B$ & Total & NO & SFOP & $\mathrm{RC}$ & $\mathrm{TI}$ & 8 \\
\hline Paciente 11 & Niño & 4 & stPNET & Parcial & $\mathrm{NO}$ & SFOP & $\mathrm{RP}$ & $M 2$ & 7 \\
\hline Paciente 12 & Niño & 3 & $M B$ & Total & NO & SFOP & $\mathrm{RC}$ & $\mathrm{T1}$ & 4 \\
\hline Paciente 13 & Niño & 5 & $M B$ & Total & sí & NO & EP & M3 & 3 \\
\hline
\end{tabular}

Edad (años); MB: meduloblastoma; stPNET: Tumor neuroectodérmico primitivo supratentorial.

SFOP: Sociedad Francesa de Oncología Pediátrica. SIOP: Sociedad Internacional de Oncología Pediátrica. CCG: Children Cancer Group.

RC: remisión completa; RP: remisión parcial; EE: enfermedad estable; EP: enfermedad progresiva.

TABLA II

Características de los pacientes con enfermedad recurrente

\begin{tabular}{|c|c|c|c|c|c|c|c|c|c|c|}
\hline & Sexo & Edad & Diagnóstico & Cirugía & Radioterapia & $\begin{array}{l}\text { Quimioterapia } \\
\text { estándar }\end{array}$ & Chang & $\begin{array}{l}\text { Situación } \\
\text { clínica }\end{array}$ & $\begin{array}{l}\text { Meses hasta } \\
\text { recaída }\end{array}$ & $\begin{array}{l}\text { Meses al } \\
\text { trasplante }\end{array}$ \\
\hline Paciente 14 & Niño & 12 & $M B$ & $\mathrm{NO}$ & $\mathrm{NO}$ & $\mathrm{NO}$ & M3 & EP & 10 & 1 \\
\hline Paciente 15 & Niño & 14 & $M B$ & Total & Sí & $\mathrm{NO}$ & M2 & $2^{\circ} \mathrm{RC}$ & 18 & 5 \\
\hline Paciente 16 & Niña & 6 & $M B$ & Total & sí & $\mathrm{NO}$ & M3 & $2^{\text {a }} \mathrm{RC}$ & 11 & 4 \\
\hline Paciente 17 & Niña & 15 & $M B$ & Parcial & $\mathrm{NO}$ & $\mathrm{NO}$ & M2 & $\mathrm{RP}$ & 24 & 2 \\
\hline Paciente 18 & Niño & 5 & stPNET & Total & sí & $\mathrm{NO}$ & $M 2$ & $2^{a} R C$ & 5 & 6 \\
\hline Paciente 19 & Niño & 15 & MB & Parcial & $\mathrm{NO}$ & $\mathrm{NO}$ & M3 & EP & 26 & 1 \\
\hline
\end{tabular}

Edad (años); MB: meduloblastoma; stPNET: Tumor neuroectodérmico primitivo supratentorial.

RC: remisión completa; RP: remisión parcial; EE: enfermedad estable; EP: enfermedad progresiva.

nal de Oncología Pediátrica (SIOP) ${ }^{7}$, y del grupo Children Cancer Group (CCG) ${ }^{8}$.

Las principales características de los pacientes de alto riesgo se encuentran reflejadas en la Tabla I.

\section{- Pacientes con enfermedad recurrente}

Los pacientes con $M B$ o stPNET recurrente recibieron tratamiento inicial con cirugía, radioterapia y quimioterapia estándar. Tras la recaída, el tratamiento consistió en máxima resección tumoral con/sin radioterapia.
Las principales características de los pacientes con enfermedad recurrente se muestran en la Tabla II.

El estadiaje de la enfermedad se realizó en todos los pacientes según la clasificación de Chang?.

Previa a la realización del trasplante, eran requeridas en los pacientes una función cardíaca normal (fracción de eyección $>45 \%$ o fracción de acortamiento $>30 \%$ mediante ecocardiografía), una función renal normal (creatinina sérica $<1 \mathrm{mg} / \mathrm{dl}$ o aclaramiento renal de creatinina $>70 \mathrm{ml} / \mathrm{min} / \mathrm{m}^{2}$ ), una función hepática normal (transaminasas en cifras inferiores a 1,5 ve- 
ces los valores normales y bilirrubina total $<1,5$ $\mathrm{mg} / \mathrm{dl}$ ), una función medular normal (hemoglobina $>10 \mathrm{~g} / \mathrm{dl}$, leucocitos totales $>3000 /$ ( L, neutrófilos absolutos totales $>1500\left(\mathrm{~L}\right.$, y plaquetas $\left.>100.000 \mathrm{~mm}^{3}\right)$ y era requerido un "score" de Lansky $>70^{10}$.

\section{- Altas dosis de quimioterapia y trasplante autólogo de progenitores hematopoyéticos de sangre periférica}

Los pacientes fueron movilizados con factores estimulantes de colonias granulocíticas (G-CSF, Neupogen ${ }^{\circledR}$, Amgen, Thousand Oaks, CA, USA) a dosis de $12 \mu \mathrm{g} / \mathrm{kg}$ dos veces al día, vía subcutánea durante 4 días consecutivos. El día siguiente de finalizada la movilización, se realizaba la leucoaféresis de grandes volúmenes de progenitores hematopoyéticos de sangre periférica, mediante un separador celular Cobe Spectra (Cobe BCT, Lakewood, CO, USA) ${ }^{11}$.

Los productos de la aféresis fueron analizados para cuantificar el número de células CD34+ mediante citometría de flujo (Epics Elite Coulter Corporation, Hialeah, Florida, USA). El producto final, conteniendo un $10 \%$ de dimetil-sulfóxido (DMSO) era congelado y conservado en nitrógeno líquido a $-196^{\circ} \mathrm{C}$.

El régimen de acondicionamiento consistió en busulfán $(4 \mathrm{mg} / \mathrm{kg} /$ día vía oral durante 4 días, desde el día -5 hasta el día -2 en 4 dosis diarias) y melfalán $1140 \mathrm{mg} / \mathrm{m}^{2} /$ día en infusión endovenosa de 5 minutos el día -1) en doce pacientes. En este grupo de pacientes, tres recibieron de manera adicional tiotepa $(250$ $\mathrm{mg} / \mathrm{m}^{2} /$ día en infusión endovenosa durante 2 días, los días -3 y -2) y cuatro topotecán $\left(2 \mathrm{mg} / \mathrm{m}^{2} /\right.$ día en infusión endovenosa de 30 minutos durante 5 días, desde el día -11 hasta el día -7).

Siete pacientes recibieron busulfán durante 4 días (4 mg/ $\mathrm{kg} /$ día vía oral, desde el día -5 hasta el día -2 en 4 dosis diarias) y tiotepa $\left(350 \mathrm{mg} / \mathrm{m}^{2} /\right.$ día durante tres días).

El día 0 fueron reinfundidos los progenitores autólogos hematopoyéticos, tras descongelarlos a $37^{\circ} \mathrm{C}$.

Desde el día +1 , todos los pacientes recibieron $G$ CSF $10 \mu \mathrm{g} / \mathrm{kg} /$ día/i.v., finalizando su administración cuando la cifra absoluta de neutrófilos (CAN) era superior a $1 \times 10^{9} / \mathrm{L}$ durante dos días consecutivos.

La toxicidad fue evaluada de acuerdo a la versión 2.0 del Instituto Nacional del Cáncer (NCl) ${ }^{12}$.

\section{- Tratamiento de soporte}

Tras la colocación de un catéter venoso central en la unidad de cuidados intensivos pediátricos (CIP), los pacientes eran aislados en habitaciones con doble puerta y sistemas de filtros de aire HEPA en la unidad de Trasplante Hematopoyético.

La profilaxis infecciosa fue realizada en todos los pacientes con cotrimoxazol.

Desde el primer día del acondicionamiento y hasta el día 0 , todos los pacientes recibieron clonazepán endovenoso para evitar las convulsiones relacionadas con el busulfán.

Las transfusiones de plaquetas se administraron para mantener recuentos plaquetares superiores a $20 \times$ $10^{9} / \mathrm{L}$ y las transfusiones de concentrados de hematíes fueron administrados para mantener un hematocrito superior al $25 \%$. Todos los hemoderivados fueron irradiados antes de la transfusión.

La antibioticoterapia de amplio espectro era iniciada cuando se documentaba temperatura $\geq 38^{\circ} \mathrm{C}$. Si el cuadro de neutropenia febril se mantenía durante más de 5 días tras el inicio de la antibioticoterapia se asociaba anfotericina $B(1 \mathrm{mg} / \mathrm{kg} /$ día).

Además, cualquier paciente con una pérdida de peso $>10 \%$, o incapacidad para la alimentación normal recibió intervención nutricional mediante soporte nutricional, enteral con sonda nasogástrica o mediante nutrición parenteral.

\section{- Definiciones}

El grupo de alto riesgo se definió como edad menor a 4 años, presencia de restos tumorales $>1.5 \mathrm{~cm}^{2}$ y confirmación mediante resonancia magnética nuclear - tomografía axial computarizada de un estadio de Chang T3b/T4 ० Ml-M48.

Para definir tumor recurrente o refractario fue necesaria la confirmación histológica o radiológica.

La extensión de la resección quirúrgica queda definida como parcial, cuando la resección tumoral es mayor del $10 \%$ pero inferior al $90 \%$; subtotal, cuando la resección tumoral es $>90 \%$ pero persiste tumor macroscópicamente y total cuando no se evidencia tumor macroscópicamente ni mediante pruebas de neuroimagen postcirugía ${ }^{8}$.

Se consideró injerto leucocitario cuando se alcanzaba CAN $>0,5 \times 10^{9} / \mathrm{L}$ durante tres días consecutivos. Se consideró injerto plaquetar cuando se alcanzaba $>20 \times 10^{9} / \mathrm{L}$ plaquetas durante tres días consecutivos sin requerimientos transfusionales.

Para determinar la respuesta terapéutica se consideraron criterios de neuroimagen. Respuesta completa (RC) es definida como la resolución de todas las lesiones y la ausencia de progresión clínica. Respuesta parcial (RP) queda definida como la reducción en $>50 \%$ en el diámetro mayor de la lesión y ausencia de progresión clínica. Enfermedad estable (EE) es definida 


\section{A. Pérez Martínez y cols.}

como la reducción de $<50 \%$ o el aumento del tamaño tumoral en $<25 \%$ sin signos clínicos de progresión y enfermedad progresiva es considerada como aumento del tamaño tumoral $>25 \%^{8}$.

\section{- Consentimiento informado}

Los padres o tutores legales de los pacientes fueron informados de los riesgos y beneficios del procedimiento, así como de otras alternativas terapéuticas. Se obtuvo consentimiento informado firmado de cada paciente.

\section{- Análisis estadístico}

La edad y datos de cinética de injerto se expresan en forma de mediana y rango. La supervivencia libre de eventos (SLE) fue definida como la presencia de progresión clínica o muerte desde el día del trasplante y hasta el día donde ocurriera tal evento. La SLE se calculó según el método de Kaplan-Meier ${ }^{13}$.

\section{Resultados}

\section{- Población}

Entre marzo 1995 y diciembre 2002, un total de 19 pacientes (12 niños y 7 niñas) con el diagnóstico de MB (14) o stPNET (5) de alto riesgo o en recaída, fueron sometidos a altas dosis de quimioterapia. La mediana de edad al diagnóstico fue de 4 años (rango 115).

Fueron clasificados 13 pacientes como de alto riesgo y 6 como enfermedad recurrente.

\section{- Pacientes de alto riesgo}

En este grupo de pacientes, 8 eran niños y 5 eran niñas. La mediana de edad fue 3 años (rango 1-14). Todos los pacientes tenían diagnóstico histológico de $M B$ (9) y stPNET (4).

El tratamiento quirúrgico se llevó a cabo en todos los pacientes, siendo la resección tumoral completa en 9 pacientes y parcial en 4.

Recibieron radioterapia 4 pacientes, todos ellos mayores de 4 años. Quimioterapia estándar fue pautada en siete pacientes (2 SIOP, 1 CCG, 4 SFOP).

La situación clínica previa a la terapia con altas dosis de quimioterapia y trasplante fue de RC en 8 pacientes, RP en 3 y EP en 2.

El régimen de acondicionamiento fue busulfán-melfalán en 8 pacientes y busulfán-tiotepa en 5; 3 pacien- tes con régimen de acondicionamiento busulfán-melfalán recibieron adicionalmente tiotepa y otros 3 recibieron topotecán.

\section{- Pacientes con recurrencia de la enfermedad}

En este grupo de pacientes 4 eran niños y 2 niñas. La mediana de edad al diagnóstico de la recaída fue de 13 años (rango 5-15).

En el momento de la recurrencia, todos los pacientes tenían neuroimagen compatible con la enfermedad y en 4 pacientes se obtuvo también documentación histológica, MB (3) y stPNET (1). La mediana de tiempo desde el diagnóstico inicial a la recaída fue de 14,5 meses (rango 5-26).

El tratamiento inicial, previo a la recurrencia, consistió en cirugía en todos los pacientes (RC en 4 pacientes, y RP en 2 de ellos), radioterapia en 3 y quimioterapia estándar en 4 pacientes (3 SIOP y 1 SFOP).

Tras la recurrencia, 5 pacientes fueron de nuevo intervenidos quirúrgicamente, alcanzando $\mathrm{RC}$ en 3 pacientes y RP en 2. Además 3 pacientes que inicialmente no recibieron radioterapia, fueron irradiados. Ningún paciente recibió quimioterapia estándar.

La situación clínica previa a las $A D Q$ fue de $R C$ en 3 pacientes, RP en 1 y EP en 2.

La mediana de tiempo desde la recurrencia hasta el trasplante fueron 3 meses (rango 1-6).

El régimen de acondicionamiento fue busulfán-melfalán en 4 pacientes y busulfán-tiotepa en $2 ; 1$ paciente con régimen de acondicionamiento busulfán-melfalán recibió adicionalmente topotecán.

\section{- Toxicidad del procedimiento y cinética de injerto leucoplaquetario}

En el día 0 se infundieron una mediana de 3,45 $(0.6-42) \times 10^{6} / \mathrm{kg}$ células de progenitores hematopoyéticos autólogos de sangre periférica. Un total de 18 pacientes recuperaron la hematopoyesis. La mediana de injerto leucocitario fue de 9 días (rango 7-12) y de injerto plaquetar 13,5 días (rango 8-60). No alcanzó injerto plaquetar 1 paciente.

Fallecieron 3 niños por toxicidad del procedimiento, 2 pacientes del grupo de alto riesgo y 1 paciente del grupo de recurrencia. La causa de la muerte fue en 2 enfermos fallo multiorgánico secundario a shock séptico y en 1 enfermo insuficiencia respiratoria por infección por citomegalovirus.

Todos los niños presentaron toxicidad hematológica grado IV, precisando una mediana de 2 unidades de concentrado de hematíes y 2 unidades de concentrado de plaquetas. 
TABLA III

\section{Acondicionamiento, cinética de injerto y toxicidades}

\begin{tabular}{llcccl}
\hline & Acondicionamiento & CD34+/kg & Injerto leucocitario & Injerto plaquetar & Toxicidades \\
\hline Paciente 1 & BU-TI & $42 \times 10^{6}$ & 7 & 15 & SEPSIS \\
Paciente 2 & BU-TI & $5,2 \times 10^{6}$ & 8 & 60 & EVOH \\
Paciente 3 & BU-TI & $2,8 \times 10^{6}$ & 9 & 25 & Convulsiones \\
Paciente 4 & BU-TI & $8,06 \times 10^{6}$ & 9 & 9 & SEPSIS/Convulsiones \\
Paciente 5 & BU-ME & $3,84 \times 10^{6}$ & 12 & 11 & EI \\
Paciente 6 & BU-TI-ME & $16,6 \times 10^{6}$ & 10 & 12 & SEPSIS \\
Paciente 7 & BU-TI-ME & $24,8 \times 10^{6}$ & 9 & 10 & - \\
Paciente 8 & BU-TI-ME & $3,45 \times 10^{6}$ & 11 & 19 & EI/SEPSIS \\
Paciente 9 & BU-ME-TO & $2,1 \times 10^{6}$ & 11 & 11 & EI \\
Paciente 10 & BU-ME-TO & $3,27 \times 10^{6}$ & 8 & 13 & VARICELA \\
Paciente 11 & BU-ME-TO & $2,4 \times 10^{6}$ & 10 & 8 & - \\
Paciente 12 & BU-ME & $13,5 \times 10^{6}$ & 11 & 14 & INFECCIÓN CMV \\
Paciente 13 & BU-TI & $14,6 \times 10^{6}$ & 7 & 15 & TUMOR 2 \\
Paciente 14 & BU-TI & $2,16 \times 10^{6}$ & 10 & - & EVOH \\
Paciente 15 & BU-TI & $2,22 \times 10^{6}$ & 9 & 9 & SEPSIS \\
Paciente 16 & BU-ME & $0,6 \times 10^{6}$ & 11 & 15 & SEPSIS \\
Paciente 17 & BU-ME & $1,26 \times 10^{6}$ & 9 & 19 & SEPSIS \\
Paciente 18 & BU-ME & $1,86 \times 10^{6}$ & 9 & 10 & - \\
Paciente 19 & BU-ME-TO & $7,3 \times 10^{6}$ & 10 & 14 & EI \\
\hline
\end{tabular}

BU-TI-ME: busulfán-tiotepa-melfalán; BU-ME: busulfán-melfalán; BU-TO-ME: busulfán-topotecán-melfalán; Injerto (días). El: enfermedad del injerto; CMV: citomegalovirus; EVOH: enfermedad venooclusiva hepática.

Todos los niños presentaron episodios de neutropenia febril que precisaron tratamiento antibiótico de amplio espectro; 1 enfermo presentó un exantema vesiculoso polimorfo característico de primoinfección por el virus varicela zóster (varicela), y 8 niños presentaron infecciones relacionadas con el catéter por estafilococo coagulasa negativo.

La toxicidad gastrointestinal fue de grado IV en todos los pacientes, precisando perfusión intravenosa de opioides para el control del dolor. Hasta 15 niños precisaron soporte nutricional en forma de nutrición parenteral con una mediana de duración de 8 días (940).

Un total de 4 pacientes presentaron síndrome del injerto precisando altas dosis de esteroides para resolver el cuadro ${ }^{14}$. Además, 2 enfermos desarrollaron enfermedad venooclusiva hepática que se resolvió con tratamiento de soporte semanas más tarde.

Neurotoxicidad grado IV en forma de convulsiones, probablemente relacionadas con el busulfán, aconteció en 2 niños.

Seis años tras el trasplante, 1 niño desarrolló un fibrohistiocitoma de hueso de localización mandibular.

La toxicidad y cinética de injerto se muestran en la Tabla III.

\section{- Respuesta terapéutica al trasplante y supervivencia}

El tumor reapareció en 7 enfermos tras una mediana de 5 meses tras el trasplante (rango 1-25 meses), 3 pacientes del grupo de alto riesgo y 4 pacientes del grupo de recaída tumoral. Todos fallecieron.

SLE

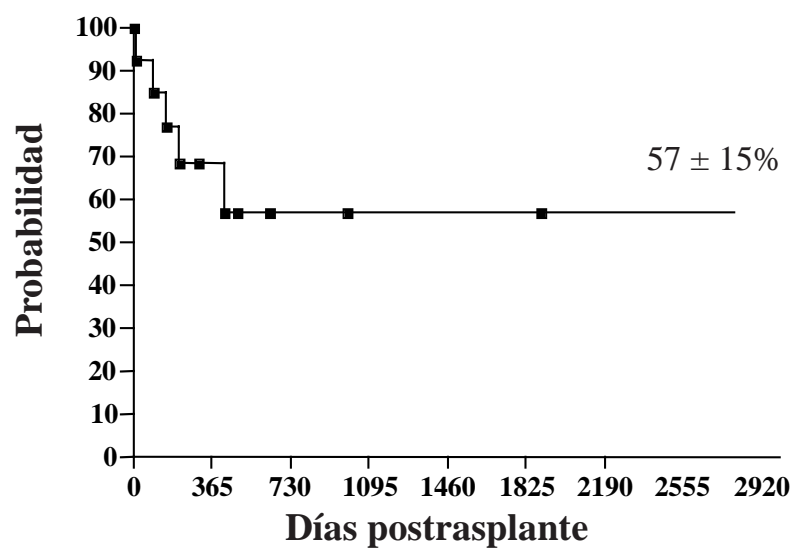

Fig. 1. Curva de supervivencia libre de eventos en los pacientes con MB/stPNET de alto riesgo. 


\section{A. Pérez Martínez y cols.}

TABLA IV

\section{Evolución y seguimiento}

\begin{tabular}{lllcr}
\hline & $\begin{array}{l}\text { Situación clínica } \\
\text { al trasplante }\end{array}$ & $\begin{array}{l}\text { Situación clínica } \\
\text { pos trasplante }\end{array}$ & $\begin{array}{c}\text { Seguimiento desde } \\
\text { el trasplante (días) }\end{array}$ & $\begin{array}{c}\text { Seguimiento desde } \\
\text { el diagnóstico (días) }\end{array}$ \\
\hline Paciente 1 & EP & MT & 18 & 120 \\
Paciente 2 & RC & RC/VIVO & 1890 & 1950 \\
Paciente 3 & RC & MEP & 210 & 300 \\
Paciente 4 & RP & MEP & 130 & 210 \\
Paciente 5 & RP & RC/VIVO & 990 & 1020 \\
Paciente 6 & RC & MEP & 428 & 1080 \\
Paciente 7 & RC & RC/VIVO & 630 & 780 \\
Paciente 8 & RC & RC/VIVO & 480 & 720 \\
Paciente 9 & RC & RC/VIVO & 150 & 720 \\
Paciente 10 & RC & RC/VIVO & 300 & 720 \\
Paciente 11 & RP & RP/VIVO & 300 & 680 \\
Paciente 12 & RC & MT & 90 & 240 \\
Paciente 13 & EP & RC/VIVO & 2790 & 2890 \\
Paciente 14 & EP & MEP & 15 & 360 \\
Paciente 15 & 2-RC & MEP & 60 & 720 \\
Paciente 16 & 2aRC & MEP & 150 & 1050 \\
Paciente 17 & RP & MEP & 150 & 1050 \\
Paciente 18 & 2-RC & MT & 750 & 1230 \\
Paciente 19 & EP & RC/VIVO & 420 & 1350 \\
\hline
\end{tabular}

RC: remisión completa; RP: remisión parcial; EE: enfermedad estable; EP: enfermedad progresiva MT: muerte tóxica; MEP: muerte por enfermedad progresiva.

En el momento actual, 9 niños se encuentran vivos, con una mediana de seguimiento tras el trasplante de 18 meses (5-63 meses). De estos nueve, 8 niños se encontraban en RC y 1 niño en RP. Sobreviven dentro del grupo de alto riesgo 8 enfermos y 1 paciente en el grupo de recurrencia.

La SLE estimada mediante el método de KaplanMeier con una mediana de 2 años, es de 37,67 \pm $14 \%$, en todos los pacientes. En el grupo de alto riesgo la SLE con una mediana de 2 años es de $57 \pm 15 \%$ (Fig. 1).

La evolución y el seguimiento se muestran en la Tabla IV.

\section{Discusión}

El reconocimiento de algunos factores pronósticos, fundamentalmente clínicos, ha permitido que algunos meduloblastomas sean considerados de alto riego 8,9 . Nosotros hemos incluido a nuestros pacientes según estos criterios y en un $47 \%$ (un total de 9) eran menores de 4 años. La gran mayoría de estos pacientes con diagnóstico de $\mathrm{MB} / \mathrm{stPNET}$ recurrente y de alto riesgo tienen un pronóstico muy desfavorable con el trata- miento convencional (cirugía, radioterapia y quimioterapia estándar) $)^{2,3}$

La utilización de $A D Q$ y posterior rescate hematopoyético, podría beneficiar a algunos pacientes en recaída, especialmente en los MB en los que el diagnóstico de la recaída se haga temprano y el trasplante de forma precoz ${ }^{15,16}$. Nosotros no hemos encontrado evidencia de mejoría en nuestros pacientes cuando hemos realizado $A D Q$ en recaída, permaneciendo sólo un paciente vivo.

Otra alternativa es utilizar las $A D Q$ en primera línea de terapia como tratamiento de consolidación en pacientes de alto riesgo ${ }^{17}$. Entre el grupo de alto riesgo, se encuentran los niños menores de 4 años en los que es necesario encontrar una alternativa terapéutica a la radioterapia para evitar su toxicidad', 3 . En el grupo de pacientes con $M B / s t P N E T$ de alto riesgo, en primera línea de tratamiento, hemos tratado a 13 pacientes (9 MB y 4 stPNET), 9 de ellos menores de 4 años. Se encuentran vivos 8 pacientes (6 MB y 2 stPNET), 4 de ellos menores de 4 años.

La buena situación clínica y el control de la enfermedad previa al trasplante han sido considerados como factores pronósticos favorables para las $A D Q^{7}$. En nuestra serie, observamos que 5 de los 9 
pacientes supervivientes (55\%) estaban en primera RC pretrasplante, mientras que tan sólo 3 de los 10 pacientes fallecidos $(30 \%)$, presentaban esta situación.

Actualmente, se desconoce cual es el acondicionamiento quimioterápico más apropiado para los tumores del SNC. Los regímenes basados en busulfán podrían ser efectivos en este tipo de tumores, tal como ha sido descrito en otros tumores sólidos ${ }^{18}$. La mayoría de las drogas utilizadas en el acondicionamiento son activas en todos los estadios del ciclo celular, por lo que la adición de algunas drogas específicas de ciclo, como inhibidores de las enzimas topoisomeras, podrían aumentar de manera sinérgica la actividad tumoricida $^{19}$. El MB es un tumor quimiosensible y los quimioterápicos con mayor actividad tumoricida serían drogas como los agentes alquilantes (busulfán, melfalán, tiotepa, ciclofosfamida y derivados del platino). Además, estas drogas presentan una cinética lineal en cuanto a la relación dosis-respuesta, por lo que serían fármacos de elección para la intensificación de la quimioterapia. Sin embargo, esta misma propiedad les confiere su limitación terapéutica, ya que el aumento de la dosis no sólo aumenta la respuesta sino también la toxicidad, principalmente hematopoyética $^{19}$, por lo que es necesario rescatar el sistema hematopoyético del paciente tras las altas dosis de quimioterapia. Clásicamente los precursores hematopoyéticos autólogos eran obtenidos de la médula ósea; sin embargo, actualmente la sangre periférica permite obtener precursores hematopoyéticos, originando injertos más precoces y duraderos, como ocurrió en todos nuestros casos.

La toxicidad del trasplante en pacientes con tumores del SNC es elevada, oscilando según las series entre el $2 \%$ y el $16 \% 1,3$. Este hecho ha limitado su uso terapéutico ${ }^{8}$. En nuestra experiencia, 3 pacientes (15\%) fallecieron por toxicidad del procedimiento, 2 por fallo multiorgánico secundario a shock séptico y 1 paciente por insuficiencia respiratoria secundaria a infección por citomegalovirus. La elevada toxicidad de las $A D Q$ sugiere la necesidad de indicar el procedimiento en aquellos pacientes en situación de remisión de su enfermedad y en buena situación clínica.

Tras una mediana de seguimiento de 2 años, la SLE global fue del $37,67 \pm 14 \%$, siendo más alta en el grupo de alto riesgo, $57 \pm 15 \%$, similar a la supervivencia de los $M B /$ st PNET de riesgo estándar ${ }^{5,19}$. Además, nuestros resultados son especialmente favorables en el grupo de MB/stPNET de alto riesgo y menores de 4 años, donde obtenemos un SLE del 71,43 $\pm 17 \%$, obviando la radioterapia y sus efectos desfavorables.
Nuestros resultados preliminares en los pacientes de alto riesgo coinciden con los descritos por otros grupos $^{1,3,8,20}$ y sugieren que los niños con MB/stPNET de alto riesgo, incluyendo los menores de 4 años, podrían beneficiarse de las ADQ como tratamiento de consolidación, evitando en este último grupo de edad la radioterapia y sus efectos secundarios.

\author{
Correspondencia: \\ Dr. L. Madero López \\ Servicio de Hemato-Oncología Pediátrica \\ y Trasplante Hematopoyético \\ Hospital Niño Jesús \\ Menéndez Pelayo, 65 \\ E-28009 Madrid \\ e-mail: Imadero@hnjs.insalud.es
}

\section{Bibliografía}

1. Newton H. Review of the molecular genetics and chemotherapeutic treatment of adult and paediatric meduIloblastoma. Expert Opin. Investig. Drugs. 2001; 10(12): 2089-104.

2. Dunkel IJ, Boyett JM, Yates A, Rosenblum M, Garvin JH Jr, Bostrom BC, et al. High-dose carboplatin, thiotepa, and etoposide with autologous stem cell rescue for patients with recurrent medulloblastoma. J Clin Oncol 1998; 16:222-8.

3. Mason WP, Grovas A, Halpern S, Dunkel IJ, Garvin J, Heller $G$, et al. Intensive chemotherapy and bone marrow rescue for young children with newly diagnosed malignant brain tumors. J Clin Oncol 1998; 16:210-21.

4. Finlay JL. The role of high-dose chemotherapy and stem cell rescue in the treatment of malignant brain tumors. Bone Marrow Transplant 1996; 18 suppl. 3, S1-S5.

5. Dunkel IJ, Finlay JL. High-dose chemotherapy with autologous stem cell rescue for brain tumors. Crit Rev Oncol Hematol 2002; 41:197-204.

6. Marec-Berare $P$, Jouvet A, Thiesse $P$, Kalifa C, Koz F, Frappaz D. Supratentorial embryonal tumors in children under 5 years of age: An SFOP study of treatment with postoperative chemotherapy alone. Med Pediatr Oncol 2002; 38:83-90.

7. Kalifa C, Hartmann O, Demeoca F, Vassal G, Couanet $D$, Terrier-Lacombe MJ, et al. High-dose busulfan and thiotepa with autologous bone marrow transplantation in childhood malignant brain tumors: a phase II study. Bone Marrow Trasplant 1992; 9(4): 227-33.

8. Zeltzer P, Boyett J, Finlay J, Albright A, Rorke L, Milstein $\mathrm{J}$, et al. Metastasis stage, adjuvant treatment, and residual tumor are prognostic factors for medulloblatoma in children: conclusions from the Children's Cancer Group 921 randomized phase III study. J Clin Oncol 1999; 17: 832-45. 


\section{A. Pérez Martínez y cols.}

9. Laurent J, Chang $\mathrm{CH}$, Cohen M. A classification system for primitive neuroectodermal tumors (medulloblastoma) of the posterior fossa. Cancer 1985; 56:1807-9.

10. Lansky SB, List MA, Lansky LL, Ritter-Sterr C, Miller D. The measurement of performance in childhood cancer patients. Cancer 1987; 60:1651-6.

11. Sevilla J, González-Vicent M, Madero L, García-Sánchez F, Díaz MA. Granulocyte colony-stimulating factor alone at $12 \mathrm{microg} / \mathrm{kg}$ twice a day for 4 days for peripheral blood progenitor cell priming in pediatric patients. Bone Marrow Transplant 2002; 30:417-20.

12. Trotti A, Byhardt R, Stetz J, Gwede C, Corn B, Fu K, et al. Common toxicity criteria: version 2.0. an improved reference for grading the acute effects of cancer treatment: impact of radiotherapy. Int J Radiat Oncol Biol Phys 2000; 47(1):13-47.

13. Kaplan EL, Meier P. Non-parametric estimation from incomplete observations. J Am Stat Assoc 1958; 3:45781.

14. Madero L, Vicent MG, Sevilla J, Prudencio M, Rodríguez $F$, Díaz MA. Engraftment syndrome in children undergoing autologous peripheral blood progenitor cell transplantation. Bone Marrow Transplant 2002; 30:355-8.

15. Guruangan S, Dunkel IJ, Goldman S, Garvin JH, Rosenblum $M$, Boyett JM, et al. Myeloablative chemotherapy with autologous bone marow rescue in young children with recurrent malignant brain tumors. J Clin Oncol 1998; 16(7):2486-93.

16. Zia MI, Forsyth P, Chaudhry A, Russell J, Stewart DA. Possible benefits of high-dose chemotherapy and autologous stem cell transplantation for adults with recurrent medulloblastoma. Bone Marrow Transplant 2002; 30:565-9.

17. Strother D, Ashley D, Kellie SJ, Patel A, Jones-Wallace $D$, Thompson $S$, et al. Feasibility of four consecutive high-dose chemotherapy cycles with stem-cell rescue for patients with newly diagnosed medulloblastoma or supratentorial primitive neuroectodermal tumor after craniospinal radiotherapy: results of a collaborative study. J Clin Oncol 2001; 10:2696-704.

18. Díaz MA, Vicent MG, Madero L. High-dose busulfan/ melphalan as conditioning for autologous PBPC transplantation in pediatric patients with solid tumors. Bone Marrow Transplant 1999; 11:1157-9.

19. Kalifa C, Valteau D, Pizer B, Vassal G, Grill J, Hartmann $\mathrm{O}$. High-dose chemotherapy in childhood brain tumours. Child's Nerv Syst 1999; 15:498-505.

20. Graham ML, Herndon JE 2nd, Casey JR, Chaffee S, Ciocci GH, Krischer JP, et al. High-dose chemotherapy with autologous stem-cell rescue in patients with recurrent and high risk pediatric brain tumors. J Clin Oncol 1997; 15:1814-23. 